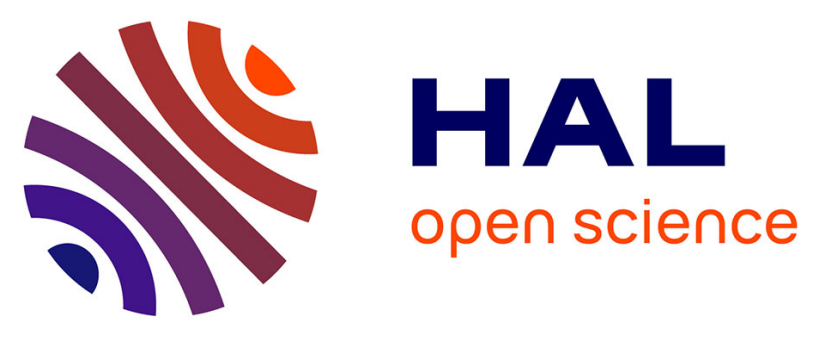

\title{
Haptic Training in a Virtual Environment to Train Cognitive Functions of Medical Students: Work in Progress
}

Nemanja Babic, Charles Barnouin, Benjamin de Witte, Arnaud Lelevé, Richard Moreau, Minh Tu Pham, Xavier Martin

\section{To cite this version:}

Nemanja Babic, Charles Barnouin, Benjamin de Witte, Arnaud Lelevé, Richard Moreau, et al.. Haptic Training in a Virtual Environment to Train Cognitive Functions of Medical Students: Work in Progress. First International Conference on Smart Multimedia, Aug 2018, Toulon, France. pp.110-120, 10.1007/978-3-030-04375-9_10. hal-01862904

\section{HAL Id: hal-01862904 https://hal.science/hal-01862904}

Submitted on 7 Jan 2019

HAL is a multi-disciplinary open access archive for the deposit and dissemination of scientific research documents, whether they are published or not. The documents may come from teaching and research institutions in France or abroad, or from public or private research centers.
L'archive ouverte pluridisciplinaire HAL, est destinée au dépôt et à la diffusion de documents scientifiques de niveau recherche, publiés ou non, émanant des établissements d'enseignement et de recherche français ou étrangers, des laboratoires publics ou privés. 


\title{
Haptic Training in a Virtual Environment to Train Cognitive Functions of Medical Students: Work in Progress
}

\author{
Nemanja Babic ${ }^{1}$, Charles Barnouin ${ }^{2}$, Benjamin De Witte ${ }^{3}$, Arnaud Lelevé ${ }^{1}$, \\ Richard Moreau ${ }^{1}$, Minh Tu Pham ${ }^{1}$, and Xavier Martin ${ }^{4}$ \\ 1 Univ Lyon, INSA Lyon, Ampère (UMR 5005), F-69621, Lyon, France, \\ arnaud.leleve@insa-lyon.fr, \\ WWW home page: http://www.ampere-lab.fr \\ 2 Univ Lyon, Univ Lyon 1, LIRIS, F-69622, Lyon, France, \\ benji.dewitte@gmail.com, \\ WWW home page: http://www.libm.fr \\ 3 Univ Lyon, Univ Lyon 1, LIBM, F-69622, Lyon, France, \\ benji.dewitte@gmail.com, \\ WWW home page: http://www.libm.fr \\ 4 Univ Lyon, Faculte de Medecine, F-69373, Lyon, France, \\ xavier.martin@chu-lyon.fr, \\ WWW home page: https://www.univ-lyon1.fr/ecole-de-chirurgie-616440.kjsp
}

\begin{abstract}
This paper introduces the development of exercises to be embedded in a lightweight laparoscopic haptic simulator to help surgeons starting their training to Minimal Invasive Surgery (MIS) gestures. These exercises were created by observing professionals in operation rooms and by isolating key gestures, which have been combined to create desired trajectories with a slow learning curve. These exercises combine memory, new gestures, new environments and new visual feedback so that the trainees' cognitive load remains low. This favors an effective training. Hence, the simulator displays a simple $3 \mathrm{D}$ virtual environment in order to focus on the gestures and trajectories, performed on an haptic device by means of real MIS tool handles. Its ludic dimension, which make it a Serious Game, should help users to make progress in their first gesture training in order to continue on more evolved medical simulators. This paper introduces the software architecture analysis and the methods used for creating the exercises.
\end{abstract}

Keywords: Medical Robotics, Haptics, Virtual Reality, Training

\section{Introduction}

The need for simulation in medical training is particularly relevant in Mini Invasive Surgery (MIS) where the operation field is not directly visible by the surgeon as his two tools operate inside the patient's body: the surgeon uses an endoscope to watch them operating. This medical approach allows the patient to recover 
faster than with open surgery but it makes the operation more difficult for the surgeon who has an indirect effect on tools (fulcrum effect) and an indirect view of his gestures. Indeed, the surgeon lacks depth perception of the operation field (through the endoscope), and is provided with a reduced visual field. Also, this technique implies to learn how to manipulate new surgical tools. Training and evaluating MIS medical gesture is therefore a difficult task.

As a result of various technological advances, the medical students are offered a more valuable and superior education than the previous generation. One of these advancements are the medical simulation platforms which train the medical staff, to decrease mistakes or accidents during medical practices (e.g. surgery). Medical simulators offer a convenient way to learn by trial and error, and are a growing solution for overcrowded medical student populations having too few opportunities to perform hands-on training during their curriculum.

Computer based simulators use haptic interfaces and calculators to provide a realistic feeling to the user and a greater number of cases without having to change worn out parts. Thanks to their software part, they offer various parameters, which enable them to reproduce an infinite number of study cases and more particularly the difficult ones. It has been validated that the skills acquired on simulators can be transferred to real operations [4] and more particularly for advanced tasks [7].

However, as these simulators tend to be very complex to use and expensive (so not sufficiently widespread), the medical training teams we work with in Universite de Lyon, identified that their students were not sufficiently efficiently trained. This is why, alongside the use of these "high resolution" simulators, their medical students undergo a training in laparoscopy using the traditional surgical "black box". The surgical "black box" is simply a portable system which reproduces the patient body by confining the operating scene into a box equipped with trocars and an endoscope, as in real MIS operations. In general, additional sensors allow the training staff to get instant visual feedback, which makes it easier to identify any mistake.

The first simulators visible in the literature provided a visual feedback of the gestures through a monitor while the users manipulated their tools in a virtual environment $[1,9]$. Later on, researches have focused on improving the quality of textures to make them more life-like [5]. As haptic feedback was missing for a full immersion in the virtual world, simulators having both visual and haptic feedback was created [10]. They are currently used in the training of medical students, for example LapSim ${ }^{\circledR}$ in Lyon's medical school. Students can then experience even rare surgical cases without jeopardizing patients' safety. These simulators are usually expensive and, as a result, cannot be freely used by every student. It hinders the continual practice necessary to learn a new medical gesture. Also, our colleagues identified an issue in the medical education field: the transition between the surgical "black box" and the medical simulators. Once completing the "black box" training, the medical students are still having trouble using the simulators. This is due to a cognitive overload. 
To fix this issue, we developed a less complex virtual environment with haptic feedback. This environment fills the cognitive gap that exists between the "black box" and the medical simulators. The virtual environment consists of simple but significant exercises to train the cognitive functions of medical students. It is expected that after this training, the students will perform with greater skills on more evolved medical simulators, such as those reviewed in [6].

This paper is organized as follows: Section 2 introduces the requirements this simulator should fulfill to be efficient in the training. Section 3 describes its software and hardware structure, and Section 4 briefly depicts the exercises.

\section{Requirements}

The main objective of the work presented in this paper is to create "low cognitive" exercises taking place in Virtual Reality and to be performed by means of two haptic devices (one per hand). Force feedback must be implemented in each exercise in order to reproduce tool-environment interactions typical in MIS operations. Three exercises should be realized:

1. An exercise involving cubes of various sizes and colors, to be simultaneously manipulated by two haptic devices, in order to construct a specific model (e.g. pyramid).

2. An exercise proposing a circuit composed of various static objects. This exercise should consist of two levels and three different views (front, side and top) each, giving a total of six different circuits that must be implemented. An evaluation of the progress is to be carried out at the end of each circuit, providing several motion quality metrics (e.g. time required, number of undesired collisions).

3. An exercise where the virtual environment features a muscular tissue and an elastic string in order to create various kinds of surgical knots. The string should be manipulated with two haptic devices if needed. The user should feel the forces when pulling the string and pushing it inside the tissue.

These specifications were provided by a group of medical doctors of Universite de Lyon, in order to train their medical students and enhance there cognitive capacity. They were reworked by the LIBM team who has strong skills in gesture training. They were then submitted and validated by the Head of Lyon Surgery Department.

\section{$3 \quad$ Simulator Structure Study}

In this paper, the investigation was mostly focused on the implementation of different exercises in a virtual world with haptic devices. Two software frameworks were selected and compared: Gazebo over ROS on the one side, and Chai3d library on the other side. Assessment functions were integrated in order to evaluate the progress of users, at the end of the second exercise. 


\subsection{Software}

Two frameworks were selected to develop such exercises necessitating a Virtual World in 3 dimensions linked to two haptic devices: Gazebo over ROS and Chai3d library.

ROS and Gazebo: The Robot Operating System (ROS $)^{5}$ is an open-source collection of software frameworks for robot software development. Indeed, it is a meta-operating system for the robot, between an operating system and a middleware. ROS can simply be illustrated as the combination of 4 parts: plumbing, tools, capabilities and ecosystem. The plumbing which is at the lowest level allows the programs to communicate between each other by providing the publishsubscribe messaging infrastructure. This set of tools offers many important features such as robot $3 \mathrm{D}$ visualization through RVIZ application, introspection, debugging, testing and more. ROS provides a collection of libraries that incorporates robot functionalities such as the advanced manipulation and path-grasp planning which enables the robot to recognize objects and react accordingly.

Gazebo $^{6}$ is a software for robot simulation. It offers a dynamic simulation environment with high-performance physics engines such as Open Dynamic Engine (ODE). Moreover, it contains an advanced 3D graphics rendering engine: OGRE. In that manner, realistic rendering of the environment is made possible such as in Fig. 1. Various sensors and plug-ins are available from laser rangefinders, cameras, contact sensors, force-torque and many more can be found in the Gazebo API. Gazebo communicates through ROS and allows the user to change or acquire various information from the simulated world. The $\mathrm{SDF}^{7}$ data provides the robots visual and physical elements to Gazebo, which then creates services for the described robot. These services can be accessed by ROS nodes using service calls.

The following list is a summary of the pros and cons of using ROS and Gazebo.

- Pros

- Possibility to build robots with multiple links and joints.

- Several degrees of freedom (DOF) for the robot.

- Force feedback (collision detection) not limited to a single point.

- Rich libraries, features, API, etc.

- Scalable: works in big projects.

- Powerful software.

- Cons

- Implementation of dual user haptic device on ROS (Linux OS).

- Modeling limitations: no pulleys, ropes, nor flexible materials.

- Focused on robot simulation; in our case, there is no robot.

\footnotetext{
5 See http://www.ros.org/

${ }^{6}$ See http://gazebosim.org/

7 SDF: Simulator Description Format
} 


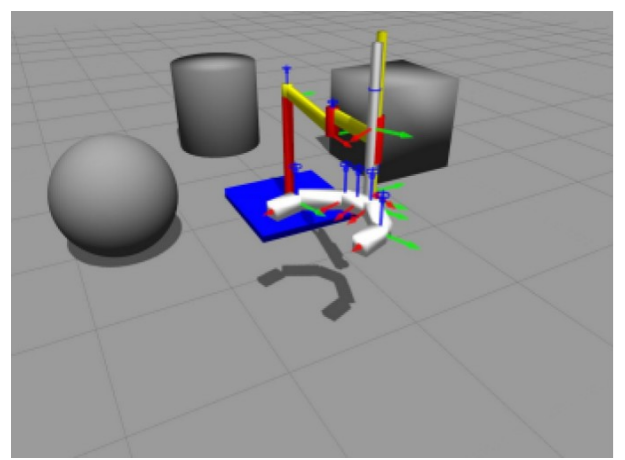

Fig. 1. Simple shapes and gripper in Gazebo

Chai3d: Chai3 $\mathrm{d}^{8}$ is an open source set of $\mathrm{C}++$ libraries for computer haptics, visualization and interactive real-time simulation. It has been designed in $\mathrm{C}++$ to make it easier and more natural for computer programmers to create applications that integrate the force feedback ability with 3D modeling.

Chai3d provides a large number of force rendering algorithms, supports several commercial devices, contains two dynamic engine for simulation and uses Open GL for graphic rendering. It is also possible to import 3D mesh files in the virtual environment. The two dynamic engine present are the ODE (Open Dynamic Engine) for rigid bodies and the GEL dynamics engine for deformable body simulation.

The Force Rendering Algorithms Module in Chai3d enables the communication between an haptic device and the virtual environment. Chai3D embeds functions to compute the impact force of collisions anywhere in space depending on the position and velocity of the device, but also to compute it when it is in contact with meshes. In other words, it computes the interaction force between the device and any virtual object. Another interesting feature that Chai3d offers is the Haptic Effects Module which enables to add viscosity to a surface to model a tissue or to increase the frequency (vibration) in order to alert the user that he is in contact with a certain object for example.

- Pros

- Simple communication with haptic devices (supports multiple devices).

- Useful set of libraries for haptic development.

- Supports flexible/deformable objects.

- Focused on haptic and visual representation.

- Cons (at the time of the realization of this project)

- Force feedback limited to the interactions between the tool tip (a simple volume) and any shape in the scene. Self-collision is not detected, which may complicate the design of exercise \#3 with suture threads to tie.

\footnotetext{
${ }^{8}$ See http://www.chai3d.org/
} 
Comparison: Chai3d was chosen taking into account the requirements for the exercises to be implemented in this project, even if ROS+Gazebo offers much more programing resources and a more evolved collision detection function than Chai3d. First, Gazebo does not support deformable objects, which is an limitation in the modeling of surgical suture, such as in the third exercise. Secondly, the intercommunication between ROS and the haptic devices is not developed as in Chai3d. The incapability of simultaneous manipulation of two haptic devices on a single machine (at the time of the start of this work, in 2015) was an issue. In turns, this limits the efficiency of some exercises to train cognitive functions. Also, Gazebo is more complex to use as it is intended for robot simulation. Indeed, developing simple exercises for cognitive training with Chai3d is much more straightforward. Moreover, Chai3d integrates at once both the haptic technology and the visual representation of objects. It supports multiple devices by detecting them automatically. The GEL Dynamics Engine is useful to model deformable objects. Also, the 2D and 3D graphical rendering and the system-level I/O is achieved with the OpenGL ${ }^{9}$ and GLUT ${ }^{10}$ libraries which are effective. Finally, our colleagues of the medical staff found it much more user friendly when interacting with the virtual environment.

Hence, Chai3d was considered a better choice to progressively prepare students for the medical simulators.

\subsection{Hardware}

The simulator reproduces a black box with real MIS tools. Yet, the tips of the tools are connected, inside the box, each one to a Geomagic Touch Haptic Device (formerly Sensable Phantom Omni) such as illustrated on the left of Fig. 2. A screen displays the virtual operating scene rendered by Chai3d, imitating the endoscope visual feedback.

\section{Exercises}

This section sums up the exercises developed during this project. Each exercise involves a different degree of cognitive difficulty.

This simulator was designed by observing surgeons in operation rooms and by isolating key gestures with the help of the Fundamentals of Laparoscopic Surgery [3]. Prono-supination, elbow flexion and extension, wrist rotations and index finger rotations were the basic anatomical movements analyzed. The exercises require the user to regularly perform these anatomical gestures. This way, they should improve their dexterity, precision, speed and other mental abilities, in the field of laparoscopic surgery. Provided MIS exercises combine memory work, gestures, and guarantee that the trainees' cognitive load remains low.

\footnotetext{
${ }^{9}$ OpenGL (Open Graphics Library) is cross-platform API for rendering 2D and 3D graphics.

10 GLUT (OpenGL Utility Toolkit) is a library of utilities for OpenGL used for systemlevel I/O.
} 

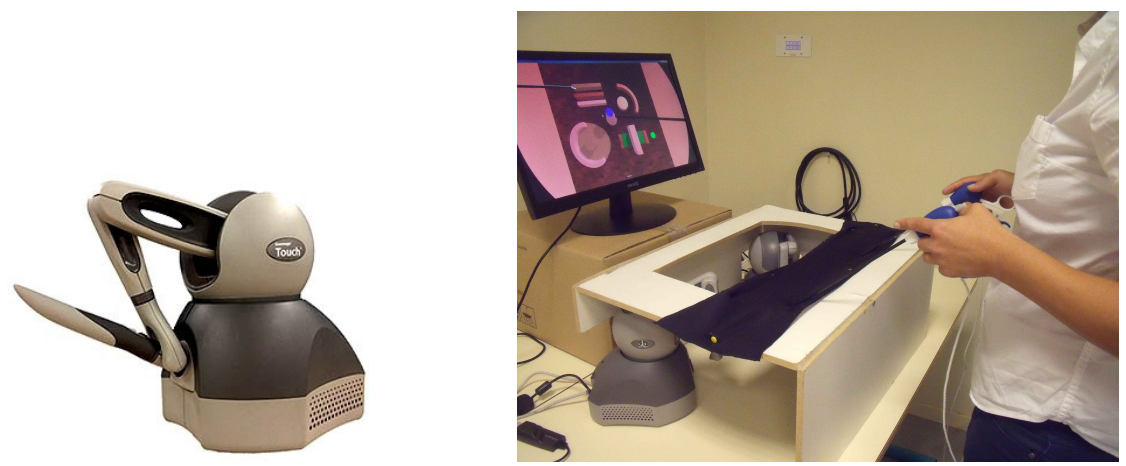

Fig. 2. On the left: a Geomagic Touch haptic device, on the right: the complete simulator with two devices hidden in the box, handled by a trainee.

Otherwise, it may negatively impact their ability to learn medical gestures [8]. Hence, it was decided to keep the exercises and the virtual environment simple, to focus on the gestures and trajectories.

\subsection{Exercise 1}

The exercise \#1 is the easiest in terms of cognitive difficulty and the objective is to build different 3D structures with cubes. It demonstrates the 3D interaction between the haptic arms - user - and the virtual environment. Fig 3 shows how cubes of different sizes and colors can be manipulated to recreate a certain structure (e.g. pyramid). In that way, medical students can train their cognitive abilities by building different structure with both hands. This exercise is the first level for the cognitive training. Many functions are integrated in this exercise:

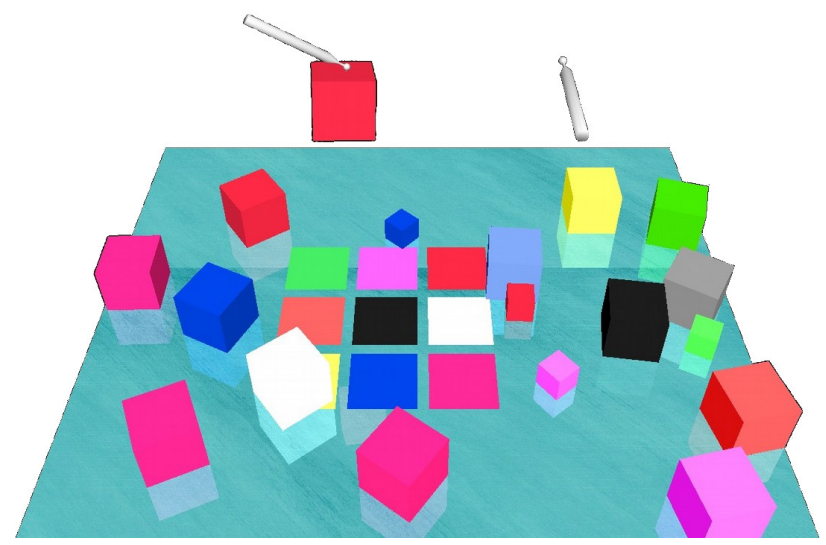

Fig. 3. Exercise 1: Dual-user haptic interaction of cubes 
- Sensation of weight for each cube.

- 3 dimensional perception (e.g. depth perception).

- Contact force at the tip of the tool.

- Collision detection and dynamics when colliding.

- The gravity can be enabled or disabled.

- Using two haptic devices simultaneously.

However, the exercise \#1 is limited with manipulations of cubes by only one tool simultaneously. Once a cube is grasped by a first tool, the second tool cannot grasp it as well.

\section{$4.2 \quad$ Exercise 2}

The Exercise \#2 is divided in two different levels and three different views (camera position). This gives a total of 6 different models to work with. The goal of the second exercise is to complete a circuit as fast as possible and without touching other objects. This exercise consists of a virtual world containing various static objects. It embeds two levels and three different views for each level. Its purpose is to train the dexterity and precision of the users when manipulating a tool. As shown in Figures 4 for level A and 5 for level B, the user must complete a circuit where the objects act like obstacles. For example, passing through each ring (level B) or doing a circular movement around the cylinder without touching it.

When starting the exercises a video clip of the circuit is shown and the students must remember the various paths (involving short term memory). In addition, the collision with each object and the time required to complete the circuit is calculated and reported once the circuit is finished.

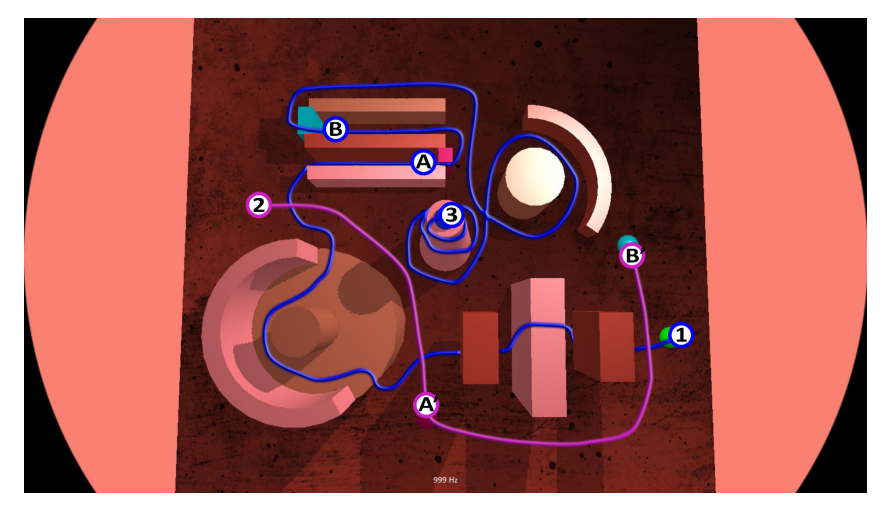

Fig. 4. Circuit to complete in Exercise \# 2 Level A, endoscopic view.

Following criteria were chosen to provide an accurate assessment: time of completion and number of collisions. However, a few points should be improved: 
- To find a solution on how to evaluate the collision with vibrating objects (which overestimates the number of collisions).

- To find a solution on how to count simultaneous collisions, e.g. when tool collides with an object while already being in contact with the ground.

- To find an additional criterion to assess the smoothness of the trajectories. An adaptation of the works introduced in [2], in the context of obstetrical gesture assessment, is in progress.

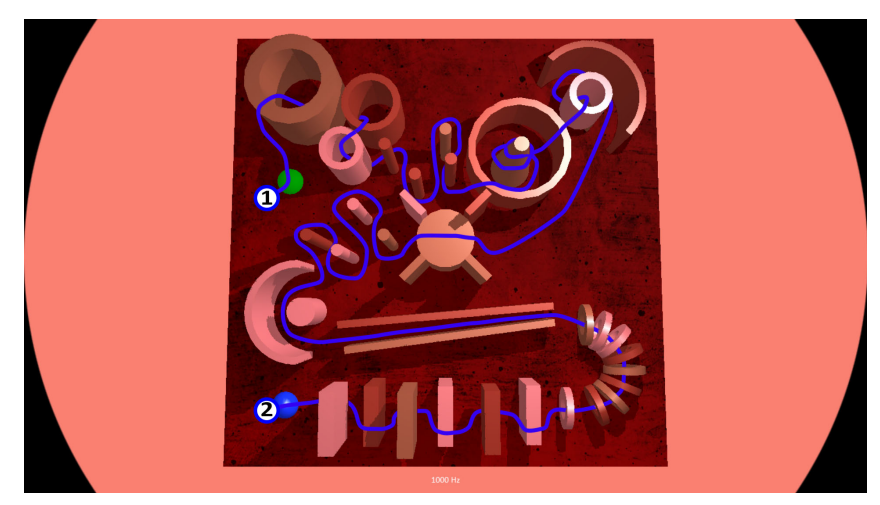

Fig. 5. Circuit to complete in Exercise \# 2 Level B, endoscopic view.

\subsection{Exercise 3}

Finally, the third exercise is considered the most difficult in terms of cognitive intensity. The purpose of Exercise \#3 is to manipulate a string in order to tie knots such as in surgical suture. The exercise \#3 incorporates a deformable string and a muscular tissue in a virtual environment. A force feedback is rendered when pulling and pushing the string. The sensation of inserting the string into the tissue is rendered as well. Figure 6 shows the skeleton of the string and the integration of dual haptic devices. Since it is not possible to "grab" the string, the program was structured such that the end of the string follows the tool sphere - when the button is pressed. Moreover, the Hooke's Law was integrated in the program to model the force feedback between the end of the string and the cursor. However, some critical points must be improved: detecting a collision between the string and a solid object, and detecting its self collision, which is mandatory to reproduce knots.

\section{Conclusion}

This paper introduces the development of exercises to be displayed to the trainees in a laparoscopic haptic simulator to help surgeons starting their training to 


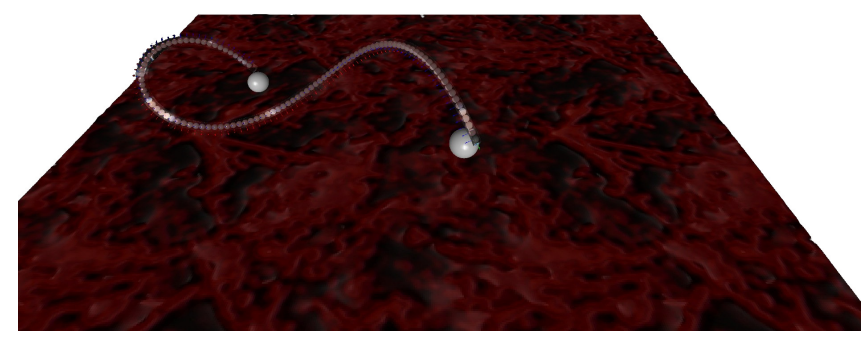

Fig. 6. Exercise \# 3: Surgical knot.

Minimal Invasive Surgery (MIS) gestures. These exercises were created by observing professionals in operation rooms and isolating key gestures which have been combined to create trajectories with a slow learning curve. This training simulator aims at helping trainees who want to acquire by themselves the necessary MIS practical skills. Its low-cost design should facilitate its spreading and availability at large scale so that students can use it freely. It will also be useful to assess their progress by means of an integrated autonomous and automatic objective assessment tool. Once sufficient skills have been acquired, trainees should be more efficient on higher fidelity simulators such as LapSim. This paper presented a description and comparison of the available softwares, besides the hardware structure of this simulator. The implementation of two exercises for cognitive training was detailed. The third one still requires work to be effective. Experimental results are being performed to determine whether a progression between novices, unexperimented interns and expert users could be detected. Also, complementary experiments are necessary to determine whether this simulator is more efficient than other existing solutions (from basic box trainer to more evolved active simulators) to train medical students on first MIS training levels.

\section{Acknowledgements}

Authors would like to thank the University of Ottawa SIRI program which helped Nemanja Babic realize this work during his internship in Ampere lab in Lyon.

\section{References}

1. Aafia Chaudhry, Christopher Sutton, Jonathan Wood, Robert Stone, and Rory McCloy. Learning rate for laparoscopic surgical skills on mist vr, a virtual reality simulator: quality of human-computer interface. Annals of the Royal College of Surgeons of England, 81(4):281, 1999.

2. Jenny Cifuentes, Pierre Boulanger, Minh Tu Pham, Richard Moreau, and Flavio Prieto. Automatic gesture analysis using constant affine velocity. In Engineering in Medicine and Biology Society (EMBC), 2014 36th Annual International Conference of the IEEE, pages 1826-1829. IEEE, 2014. 
3. Gerald M Fried. Fls assessment of competency using simulated laparoscopic tasks. Journal of Gastrointestinal Surgery, 12(2):210-212, 2008.

4. A Hyltander, E Liljegren, PH Rhodin, and H Lönroth. The transfer of basic skills learned in a laparoscopic simulator to the operating room. Surgical endoscopy, 16(9):1324-1328, 2002.

5. Doug L James and Dinesh K Pai. Dyrt: dynamic response textures for real time deformation simulation with graphics hardware. In ACM Transactions on Graphics (TOG), number 21:3, pages 582-585. ACM, 2002.

6. Danielle Julian, Alyssa Tanaka, Patricia Mattingly, Mireille Truong, Manuela Perez, and Roger Smith. A comparative analysis and guide to virtual reality robotic surgical simulators. The International Journal of Medical Robotics and Computer Assisted Surgery, 14(1):e1874-n/a, 2018. e1874 RCS-17-0115.R2.

7. L. Panait, E. Akkary, R.L. Bell, K.E. Roberts, S.J. Dudrick, and A.J. Duffy. The role of haptic feedback in laparoscopic simulation training. Journal of Surgical Research, 156(2):312-316, Oct. 2009.

8. Jeroen JG Van Merriënboer and John Sweller. Cognitive load theory in health professional education: design principles and strategies. Medical education, 44(1):8593, 2010.

9. JD Westwood, HM Hoffman, D Stredney, and SJ Weghorst. Validation of virtual reality to teach and assess psychomotor skills in laparoscopic surgery: results from randomised controlled studies using the mist vr laparoscopic simulator. Medicine Meets Virtual Reality: art, science, technology: healthcare and evolution, page 124, 1998.

10. Derek T Woodrum, Pamela B Andreatta, Rajani K Yellamanchilli, Lauren Feryus, Paul G Gauger, and Rebecca M Minter. Construct validity of the lapsim laparoscopic surgical simulator. The American Journal of Surgery, 191(1):28-32, 2006. 